\title{
Coronary disease, cardioneuropathy, and conduction system abnormalities in the cardiomyopathy of Friedreich's ataxia ${ }^{\star}$
}

\author{
THOMAS N JAMES, $\uparrow$ B WOODFIN COBBS, $\ddagger$ H CECIL COGHLAN, $\uparrow$ \\ WALTER C MCCOY, $\dagger$ CHARLES FISCH§ \\ From the $\dagger$ University of Alabama School of Medicine, Birmingham, Alabama, the $\ddagger$ Emory University \\ School of Medicine, Atlanta, Georgia, and the §University of Indiana School of Medicine, Indianapolis, \\ Indiana, USA
}

SUMMARY Abnormalities of the heart are a frequent and possibly ubiquitous problem in patient with Friedreich's ataxia, but their pathogenesis is unclear. Postmortem findings are reported from the hearts of three patients with Friedreich's ataxia who died of congestive heart failure and atrial arrhythmias. Particular attention was paid to the following: the large and small coronary arteries, the nerves and ganglia, the conduction system, and the histological and cellular features of the cardiomyopathy. There were pleomorphic nuclei and focal fibrosis and degeneration throughout each heart including the conduction system. There were distinctive abnormalities of both large and small coronary arteries, and focal degeneration of nerves and ganglia. These observations suggest a mosaic concept for the pathogenesis for the cardiomyopathy of Friedreich's ataxia that involves the interplay of molecular faults, cardiomyopathy, cardioneuropathy, and coronary disease.

In Friedreich's original description of the heritable ataxia that now bears his name he reported that five of his six cases showed clinical evidence of heart disease. ${ }^{1}$ Although the existence of such heart disease has been recognised for many years-and despite Friedreich's own experience-it has only recently come to be widely appreciated how often the heart is abnormal in these ataxic individuals. Current estimates in two large series of carefully studied cases suggest that between $90 \%$ and $100 \%$ of patients with definite Friedreich's ataxia will have some demonstrable abnormality of the heart, whether or not any clinical cardiac manifestations have yet appeared. ${ }^{23}$

Despite the continuing investigations of heart disease of Friedreich's ataxia, ${ }^{4-31}$ uncertainty about its pathogenesis remains. It is known that affected individuals may develop cardiac hypertrophy of either

Requests for reprints to Dr Thomas N James, Callaway Laboratory of the Department of Medicine, University of Alabama Medical Center, Birmingham, Alabama 35294, USA.

*Presented in part at the annual meeting of the Association of University Cardiologists on 17 January 1986 in New Orleans.

Accepted for publication 11 December 1986 the symmetrical or asymmetrical type and with or without dilatation, that they eventually have progressively increasing congestive heart failure which is often the cause of death, and that their clinical course is characterised by the occurrence of $\dot{0}$ arrhythmias and various other forms of electrical instability of the heart. But these clinical descriptive 8 features are of little help in determining the fundamental basis of the heart disease; and without that $\mathrm{O}$ knowledge it will continue to be difficult to determine prognosis or to develop optimal forms of treatment.

Previous studies have unequivocally shown that the small coronary arteries are abnormal in patients who have cardiac disease and Friedreich's $\tilde{\omega}$ ataxia $^{58-12}$; but the functional significance of this finding has been challenged, particularly by Hewer ${ }^{11} O$ whose findings have been widely quoted in virtually $\mathbb{D}$ all subsequent reviews. In the present report we de- $\rightarrow$ scribe our own findings in three patients, which sup- $T$ port the importance of small coronary artery disease, $\stackrel{0}{\mathbb{D}}$ and show why we believe that Hewer's personal observations do in fact accord our findings.

A second cardiac abnormality originally described 0 by Dorothy Russell, ${ }^{5}$ who may also have been the $\delta$ 
first to call attention to the coronary disease in her case 3 , is a form of myocarditis which we believe is better regarded as a form of cardiomyopathy. While the cardiomyopathy is undoubtedly influenced by disease of the small coronary vessels, cardiomyopathy seems unlikely to be explained entirely by such disease.

The third abnormality that is abundantly present is cardioneuropathy, which we believe is described for the first time in this report. A fourth component of our study deals with the conduction system of the heart, abnormalities of which help to explain the frequent manifestations of cardiac electrical instability in these patients. We believe that interdependent relations of all these factors form the basis for a mosaic concept of the pathogenesis of heart disease in patients with Friedreich's ataxia.

\section{Clinical observations}

\section{CASE 1}

This twenty year old white man was first found to have Friedreich's ataxia at about the age of eight, and within two years intermittent heart failure that was responsive to treatment with conservative measures developed. Over the next decade his cardiac failure progressed only gradually, although several bouts were transiently severe. These were often associated with episodes of pulmonary infection. He was usually in sinus rhythm with multiple atrial extrasystoles, but several bouts of atrial fibrillation were documented, especially in his last year of life. The terminal illness was characterised by an abrupt onset of severe hypotension accompanied by cyanosis and dyspnoea. It was briefly and incompletely responsive to treatment with digitalis, diuretics, and similar measures. He died eight days after the onset of hypotension.

\section{CASE 2}

A twenty two year old white man was first diagnosed as having Friedreich's ataxia at the age of six. In the last five years of his life he had recurring bouts of atrial flutter and fibrillation that were eventually associated with steadily worsening congestive heart failure. Several episodes of pulmonary infection complicated cardiac disease. During this time he complained of a dull substernal non-radiating pain, which lasted 10 to 15 minutes. It was often produced by emotional stress. He was too disabled to undertake any activity that was physically taxing. His final admission was for severe pulmonary congestion which became intractable and led to cyanosis and coma. He died the next day.

CASE 3

This twenty year old white man had Friedreich's ataxia with progressive cardiac failure for five years. There were intermittent bouts of atrial fibrillation. Recurring episodes of pulmonary infection aggravated the cardiac insufficiency. During his last admission the congestive failure was more severe and there was atrial flutter with partial heart block. He died quietly on the fifth hospital day. Other details of this case have been reported before. ${ }^{8}$ It is included here after further assessment of the coronary disease and a special study of the nerves and ganglia of the heart, which were not a subject of the previous study.

\section{Morphological observations}

In all three cases the heart was generally enlarged but not much dilated. Gross heart weights for cases 1, 2, and 3 were $470 \mathrm{~g}, 495 \mathrm{~g}$, and $550 \mathrm{~g}$ respectively. All three cases had extensive pulmonary and other visceral congestion, varying amounts of bronchopneumonia, and histological evidence of spinocerebellar degeneration. Within the hearts there was no valve disease, the interatrial and interventricular septa were intact, and there were no gross

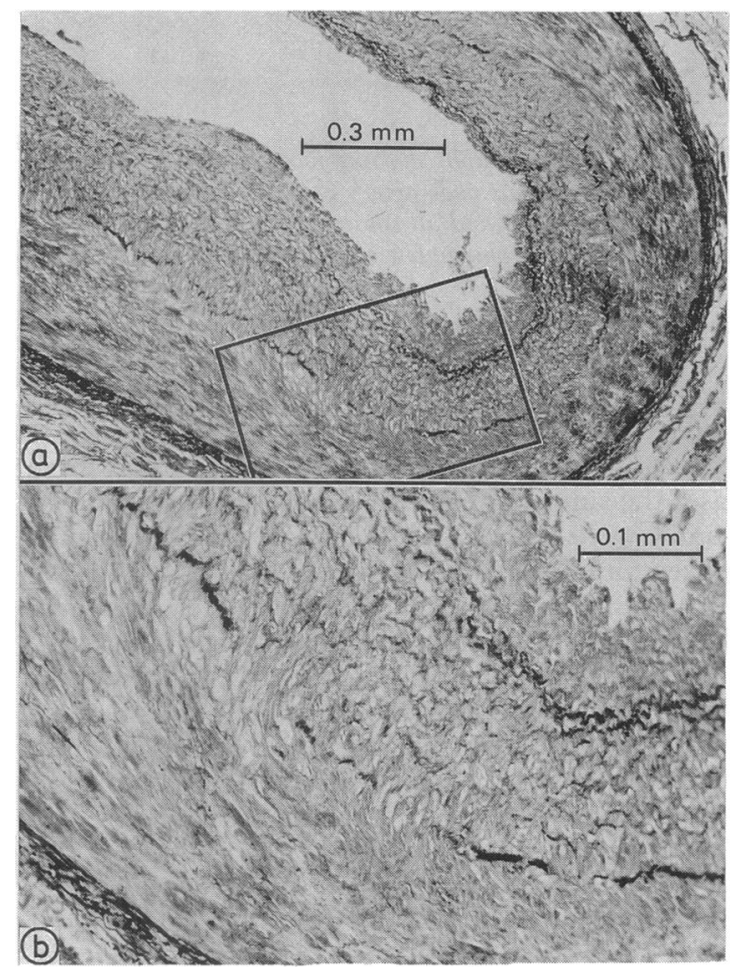

Fig 1 Photomicrographs showing focal fibromuscular dysplasia of the main right coronary artery shown at two magnifications ( $a$ and $b$ ). Verhoeff-van Gieson elastic stain. All magnifications are indicated by reference bars. 


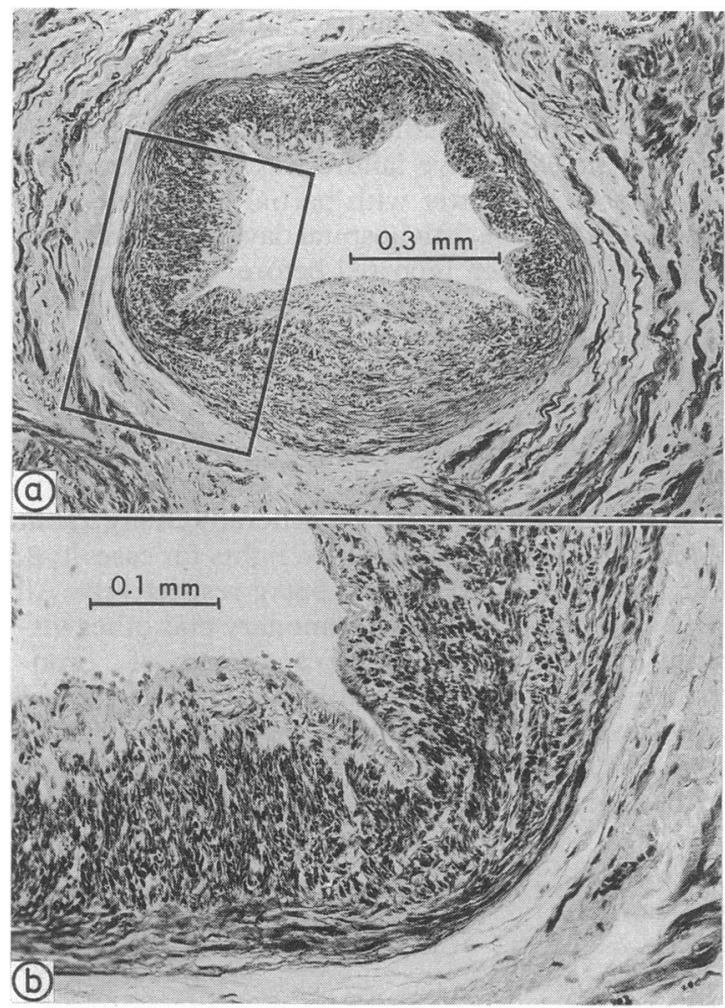

Fig 2 Photomicrographs showing focal fibromuscular dysplasia of the sinus node artery at two magnifications (a and $b$ ). This section and all subsequent sections are stained with Goldner trichrome unless stated otherwise.

anomalies or malformations. In each case the pericardium was slightly fibrotic, especially in cases 1 and 3, but there was no active pericarditis and there were no important adhesions.

Because there was atrial arrhythmias in all three cases the sinus node, atrioventricular node, and $\mathrm{His}$ bundle were examined. ${ }^{32} 33$ In addition to tissue removed for examination of the conduction system, we also examined four slices of left ventricle (minimal size 15 by $40 \mathrm{~mm}$ ), four of right ventricle (at least 10 by $40 \mathrm{~mm}$ ), and two of left atrium (at least 5 by 25 $\mathrm{mm}$ ). The sinus node sections included considerable amounts of right atrium. At least 10 serial $2 \mathrm{~mm}$ slices of sinus node were available from each case. The sections of atrioventricular node and $\mathrm{His}$ bundle included at least $2 \mathrm{~cm}$ of both atrial and ventricular septal tissue, and for each case there were at least eight $2 \mathrm{~mm}$ slices cut serially from this region. From each of these tissue slices at least 10 serial sections of $8 \mu \mathrm{m}$ thickness were prepared with the Goldner trichrome stain; additional sections were stained with the periodic acid Schiff, Verhoeff-van Gieson elastic, and congo red methods.

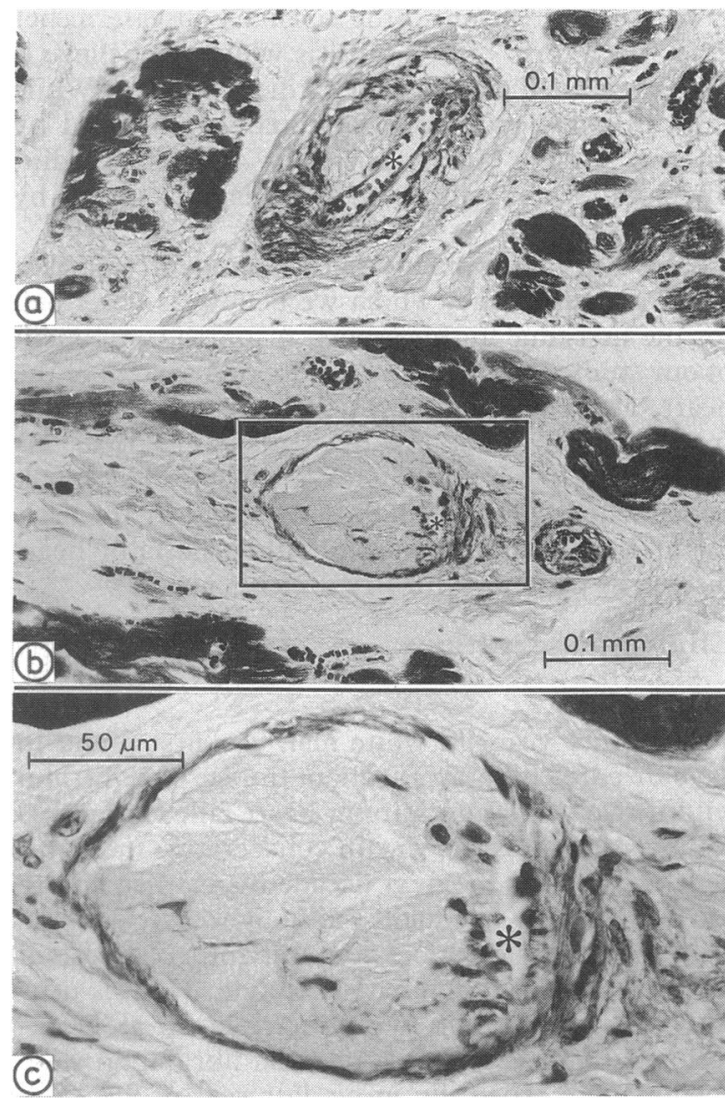

Fig 3 Photomicrographs showing abnormalities of the small coronary arteries in branches of the atrioventricular node artery. The lumen (asterisk) in (a) is narrowed by an intramural amorphous deposit and there is degeneration of the tunica media. (b) Only thin tunica media remains, with an even larger amorphous intramural deposit in the artery. This is shown at higher magnification in (c). The tiny lumen remaining is indicated by an asterisk.

As part of the light microscopic examinations at least 400 colour photomicrographs were prepared of each of the three cases. Study of both these and the histological slides showed that the myocardial, neural, and arterial lesions were similar in nature and distribution in each case. Black and white photomicrographs from case 1 were then prepared; they are representative of all three cases. The important categories of abnormalities are the coronary disease, the cardioneuropathy, and the conduction system as well as a probably separate myocytopathy of the heart.

CORONARY DISEASE

In the previous report of case 3 an abnormality of one 


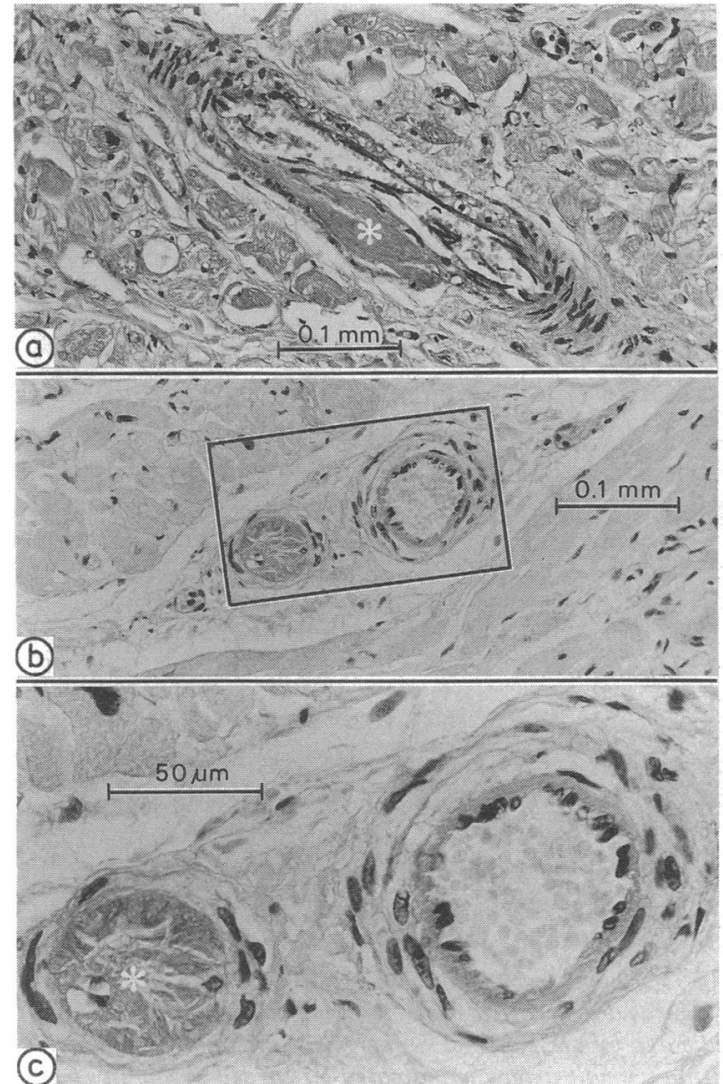

Fig 4 Photomicrographs showing staining of the amorphous intramural deposits in small coronary arteries by periodic acid-Schiff. In the tangentially cut artery (a) the deposit is marked with a white asterisk. There is one patent artery and one that is nearly occluded (b). These are shown at higher magnification (c). The dark

Schiff-positive material is marked with a white asterisk.

large coronary branch was briefly mentioned. This result, the description by Dorothy Russell of constricting atheromas in the large and small coronary arteries of her case 3 (a 21 year old man) and similar findings by Lamarche $e t a l,{ }^{12}$ and the recent report of clinically important coronary spasm in Friedreich's cardiomyopathy ${ }^{29}$ prompted a careful examination of the large coronary arteries of our three cases. In addition to preparing cross sections at intervals of no more than $5 \mathrm{~mm}$ within all three major coronary trunks for gross examination, we examined histological sections taken from at least four equidistant sites within the three main coronary arteries, beginning at the origin of each artery. In all three hearts we found three or more sites of narrowing without occlusion of two or more major coronary trunks (that is those that were at least $1.5 \mathrm{~mm}$ in

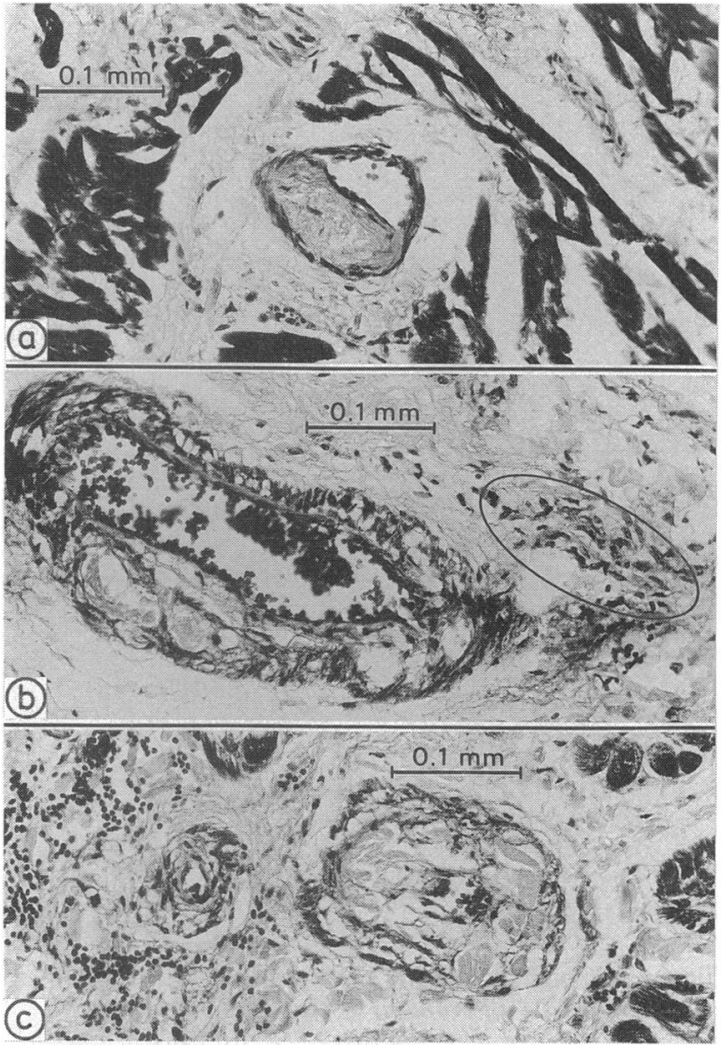

Fig 5 Photomicrographs showing material stained with periodic acid-Schiff stain without much associated degeneration in some vessels (a) and extensive association with medial degeneration, which is often but not always cystic in nature ( $b$ and $c)$, in others. $A$ small degenerating nerve is circled in (b). The artery in $(a)$ is in the interventricular septum, the one in (b) is in the interatrial septum, and the one in (c) is near the sinus node.

diameter). At no point was there any coronary thrombosis nor any typical atheroma formation nor endothelial ulceration or fissuring. Although some stenotic lesions showed intimal proliferation, the principal cause of narrowing was focal fibromuscular dysplasia (figs 1 and 2). None of the large coronary arteries showed the special Schiff positive intramural lesion often found in small coronary arteries. ${ }^{8}$

A wide range of histological abnormalities have been reported in stenosis of the small coronary arteries. ${ }^{8}$ All types of these lesions were focal in distribution; for example serial examination of sections from the same small artery always disclosed some normal segments even when there was indisputable disease in another area (figs 3-6). The following histological abnormalities were found: medial degeneration and fibrosis; intimal proliferation; focal 


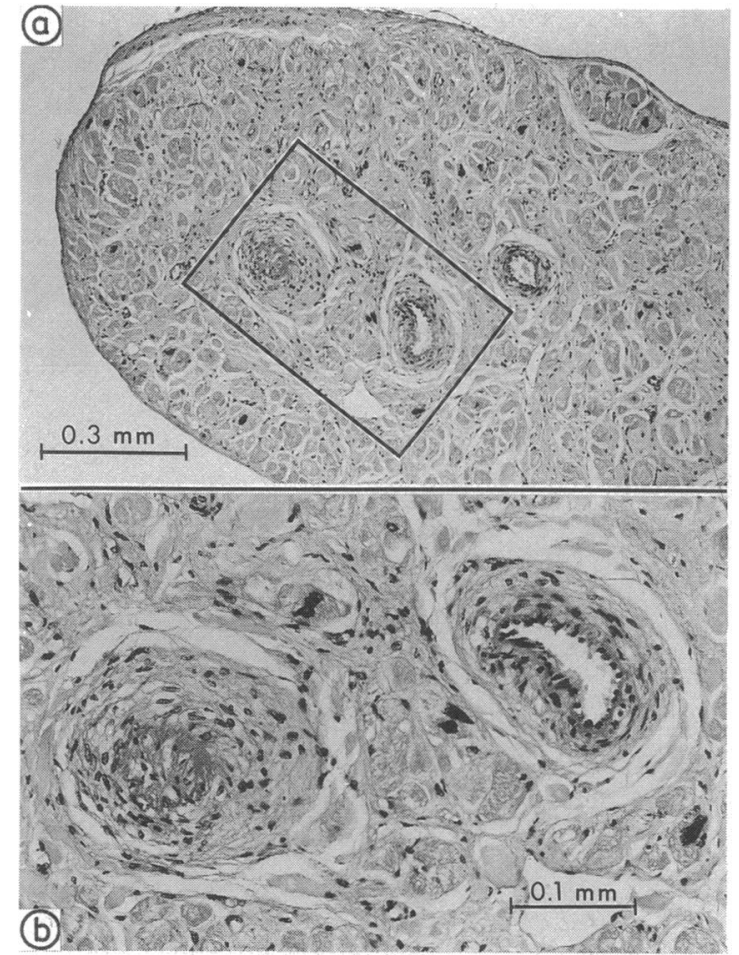

Fig 6 Photomicrographs of three small arteries in the crista terminalis, all branches of the sinus node artery, showing a range of abnormalities. The two more distinctly abnormal ones are boxed in (a) and seen at higher magnification in (b). A subintimal deposit of material stained by periodic acid-Schiff reagent is evident in the artery to the left in (b) and smaller amounts are seen scattered in a neighbouring artery.

fibromuscular dysplasia; and either subintimal or medial deposition of an amorphous material stained by periodic acid-Schiff method. The last three abnormalities all produced luminal narrowing. These four histological abnormalities were demonstrable in all of the three cases, and sometimes in different segments of the same artery (for example in the sinus node artery, atrioventricular node artery, or the long branches in the interventricular septum). Inflammation was rarely seen in the arteries and when it was it was only slight.

So that we could compare our results with those of Hewer ${ }^{11}$ we examined at least 100 cross sections of coronary arteries from subserial sections of the interatrial and interventricular septa, like those that are routinely prepared for study of the atrioventricular node and His bundle. When we used Hewer's criterion of $>50 \%$ narrowing of an arterial lumen as being "functionally significant" (a point which we dispute), we found, as he did, approximately $9 \%$ of such narrowings. In these three cases lesions causing

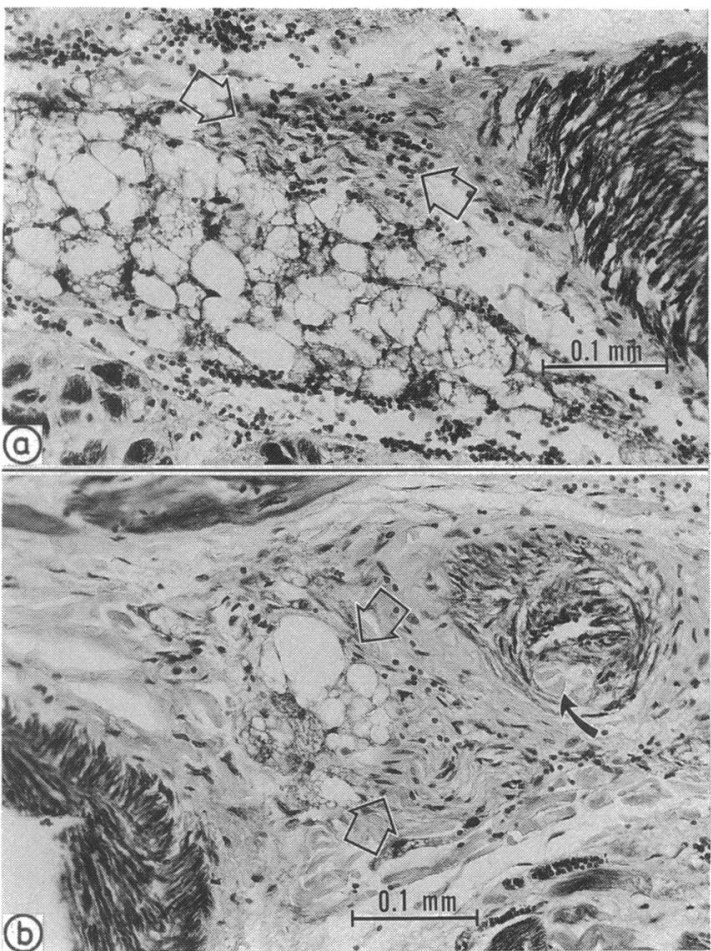

Fig 7 Photomicrographs showing two examples ( $a$ and $b$ ) of neural degeneration near the sinus node. Neural tissue is indicated by open arrows. Amorphous intramural deposit within a small artery is indicated by curved arrow in (b).

$>50 \%$ narrowing were found in $5 \%$ to $8 \%$ of examined sections of small arteries. When 100 consecutively encountered cross sections of small coronary arteries of the left ventricular slices were examined, we found that only 3-5\% showed narrowing of $\geqslant 50 \%$. In all myocardial samples studied there were structural abnormalities of the small coronary arteries (not causing 50\% narrowing) in $35-40 \%$ of cross sections of arteries that were between 100 and $1000 \mu \mathrm{m}$ in diameter. Later we will explain why these lesions cannot be regarded as functionally insignificant.

The nature of the Schiff positive material characteristically found in the small coronary arteries of Friedreich's cardiomyopathy is not known. We wonder why others do not appear to have sought this lesion. Morphologically it resembles deposits of amyloid, but neither in the original description ${ }^{8}$ nor in specially prepared congo red stains of these three cases for the present report was there any specific staining for amyloid. Similarly, stains for fat or fibrin have been consistently negative. Small deposits of this Schiff-positive material were found rarely in a nerve or within myocytes, but the material was most 


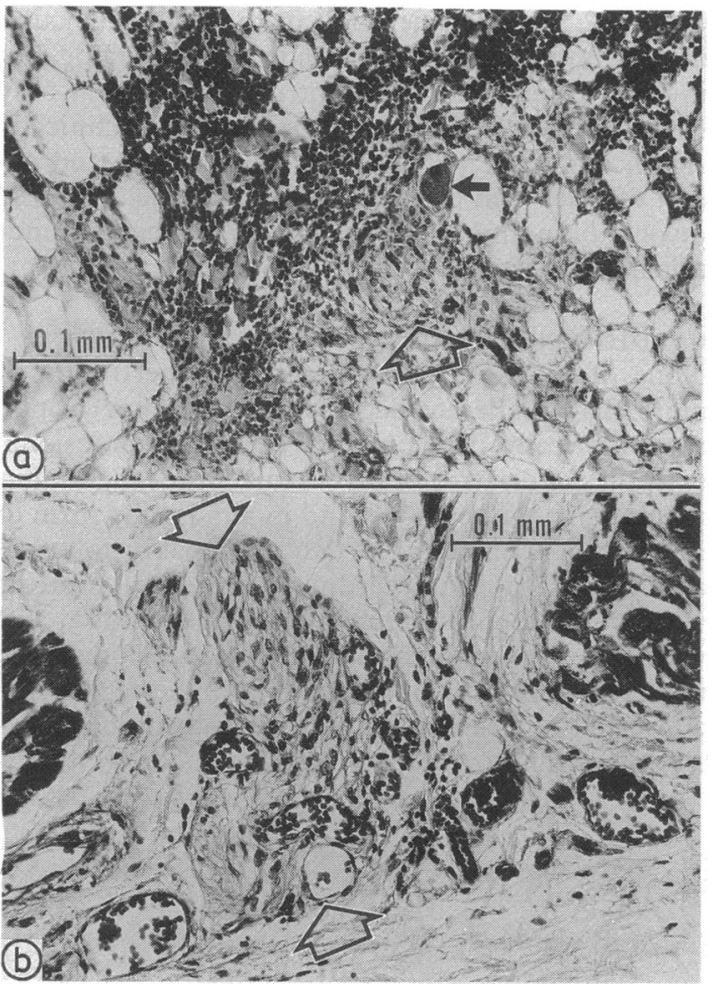

Fig 8 Photomicrographs showing haemorrhagic degeneration of a nerve (open arrow) in the sinus node (a) with one ganglion cell indicated by a black arrow. A degenerating nerve near the atrioventricular node is indicated by two open arrows (b).

often and readily demonstrable in the small coronary arteries. Dr Alexander Nadas kindly gave us the opportunity of examining sections of the original case of Nadas et al. ${ }^{6}$ The stenotic lesions in the small coronary arteries also contained Schiff positive material. As in our own cases, however, the Nadas case also had other histological lesions that narrowed the small coronary arteries but did not contain Schiff positive material.

\section{CARDIONEUROPATHY}

We especially studied nerves and ganglia in the conduction system, where they are abundant. In all three hearts there was extensive focal degeneration of the myelinated and unmyelinated nerves and the cardiac ganglia (figs 5,7 , and 8). Some of these examples were associated with a moderate degree of round cell infiltration whereas others showed principally fragmentation and necrosis without much cellular response. In addition to the neural lesions that were easily demonstrable within and near the conduction system we found similar neural abnormalities within

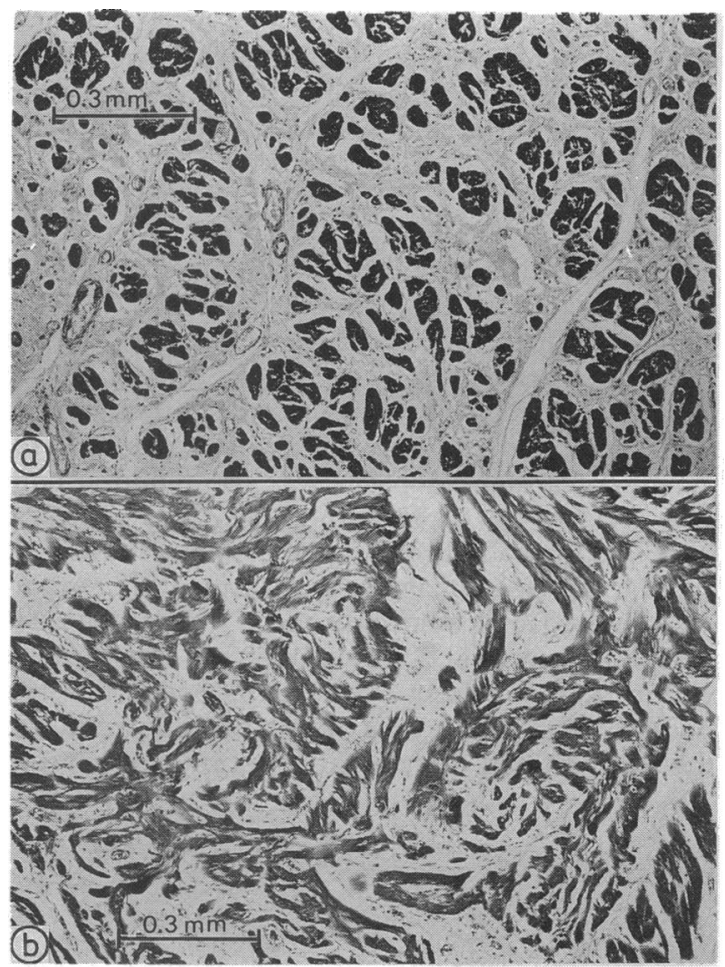

Fig 9 Photomicrographs showing focal fibrosis (a) in the posterior papillary muscle of the left ventricle. (b) Fibre disarray intermingled with some fibrosis within the interventricular septum.

both the right and left ventricular myocardium. Because we studied at least 10 serial sections from every tissue block we could be certain that the neural lesions were focal. Relatively normal nerve tissue was often present 30 or $\mathbf{4 0} \mu \mathrm{m}$ away from an extensively diseased area. Interruption of a nerve at any point can be functionally important for the entire nerve.

We did not use special stains to determine whether the observed neural lesions occurred in sympathetic or parasympathetic nerves. Special silver impregnation stains have no significant advantage over the demonstration of cardiac neural structures with the Goldner trichrome stain. ${ }^{34}$ Because most ganglia within the heart are considered to be components of the vagal system, we assume that the lesions of ganglia occurred in parasympathetic units. Local nerves were included in some regions of myocardial degeneration, but cardioneuropathy was also often found as isolated degeneration with no neighbouring myocardial disease. In cross section small coronary arteries characteristically have paired nerves flanking them, ${ }^{34}$ especially in the atria. Although there were 
juxta-arterial lesions of nerves accompanying some arterial lesions, the two abnormalities were often found separately, that is neural lesions were found away from arteries and abnormal arteries had local nerves of normal appearance.

\section{CONDUCTION SYSTEM}

There were distinct and consistent abnormalities of the conduction system in all three hearts. In each the sinus node (figs $2,5,6$, and 8 ) was more affected than the atrioventricular node or the His bundle. There were focal narrowings of local arteries both in the sinus node and the atrioventricular junctional region, and cardioneuropathy was also found at both sites. Within the three sinus nodes there was focal degeneration and fibrosis, attributable in part to the coronary disease but also separate from it. For example, the bizarre pleomorphic nuclei seen in myocytes outside the conduction system were also present within it, particularly in the sinus node. The His bundle was generally intact in all three cases and was the least affected component of conduction system.
In case 2 focal degeneration in the atrioventricular node almost equalled that seen in the sinus node, but in cases 1 and 3 the atrioventricular node was only slightly affected. Since all three cases had clinically significant atrial arrhythmias, the presence and extent of disease in the sinus node and adjacent atrial myocardium must be interpreted as being functionally important.

CARDIOMYOPATHY AND MYOCYTOPATHY

None of the three hearts showed hypertrophy of more than moderate degree, nor was hypertrophy asymmetrically distributed. There were occasional examples of disarray of myocardial fibres (fig 9), but no more than is readily found in almost any form of cardiac hypertrophy. The two principal histological abnormalities of myocardium were focal fibrosis with and without accompanying degeneration, and wide distribution of bizarrely shaped pleomorphic nuclei (figs 10 and 11) present in both the ventricular and atrial myocardium. Both the fibrotic degeneration and the bizarre pleomorphic nuclei were em-

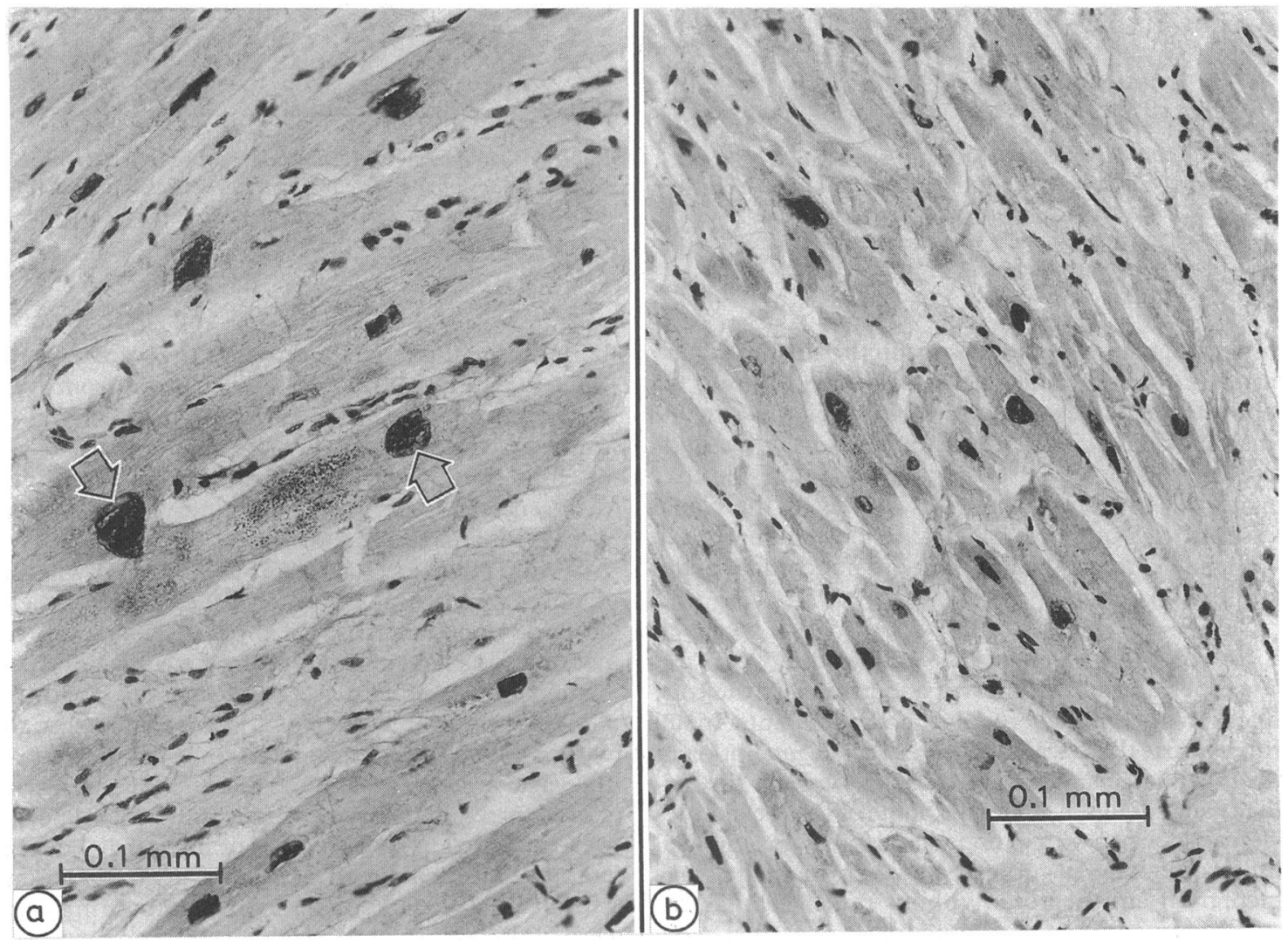

Fig 10 Photomicrographs showing pleomorphic myocardial nuclei (two indicated with open arrows in $(a)$ ) in the interventricular septum near the atrioventricular node. Periodic acid-Schiff stain. 

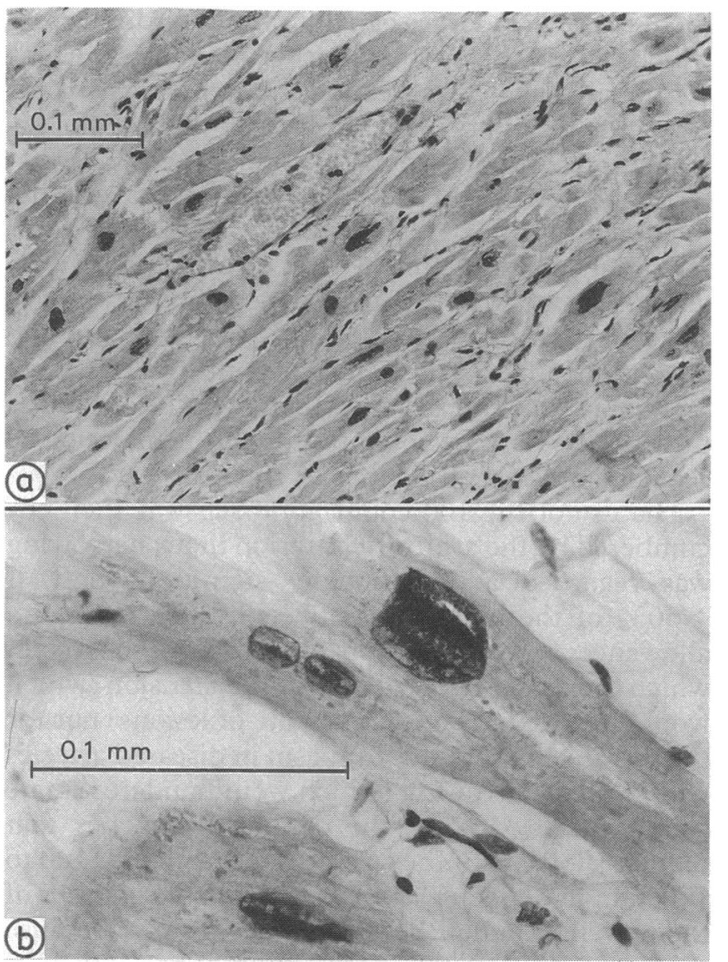

Fig 11 Photomicrographs showing other pleomorphic nuclei from the same region as in fig 10. Note the giant nucleus in (b). Periodic acid-Schiff stain.

phasised by Russell. ${ }^{5}$ Although she referred to these features collectively as myocarditis, the implication for some workers (though not her, of course) that an infection was somehow involved suggests that it would be better to use some other term. The noncommittal and non-specific term, cardiomyopathy, seems suitable, with special emphasis upon the unusual nuclear features in these cases (figs 10 and 11).

As with the cardioneuropathy, there was no consistent spatial relation between observed neural or arterial lesions and those of the myocardium. There can be little doubt, however, that some and possibly much of the focal myocardial degeneration was causally related and attributable to some of the focal arterial lesions that severely narrowed or obliterated those arteries. An assessment of the functional significance of the cardioneuropathy is complicated by the fact that neural abnormalities in the extracardiac nerves or the spinal cord or brain could also have profound effects upon the myocardium.

\section{Discussion}

The findings in these three cases, the clinical features of which are representative of most reported experi- ences with Friedreich's cardiomyopathy, lead to a reasonable multicomponent concept of the pathogenesis of the disease. These components include disease of both the large and small coronary arteries but especially the small ones, cardioneuropathy (nerves and ganglia), and cardiomyopathy (focal fibrosis plus intracellular, especially nuclear, abnormalities of myocytes). In the conduction system these three components could combine to produce the cardiac electrical instability found in Friedreich's ataxia. These components suggest why the heart becomes enlarged and eventually fails, why arrhythmias and conduction disturbances are so common, and why there is often an inexorably downhill clinical course with death within the first two or three decades.

\section{CORONARY DISEASE}

For many years coronary disease has been suspected in patients with Friedreich's ataxia for some obvious reasons. They often complain of chest pains. Their electrocardiograms often show widespread inversion of $T$ waves. They often have palpitation, arrhythmias, syncope, and sudden death. None the less, there is not simply a continuing dispute as to the importance of coronary disease in Friedreich's cardiomyopathy-most reports and reviews of the subject dismiss the coronary lesions as not functionally significant, largely on the basis of Hewer's interpretations. ${ }^{11}$ The occurrence of lesions in the small coronary arteries, which Hewer and many others have now documented, is not disputed. ${ }^{568-1012}$ Two factors will help clarify this matter: they are an examination of the history of such studies and a specific critique of the Hewer study.

Although she did not emphasise the finding, Russell found atheroma in two of her four cases and she commented that in one (her case 3 ) both the large coronary arteries and their branches were constricted but not occluded. ${ }^{5}$ Whether these lesions were caused by atheroma or some other narrowing process is uncertain. Their occurrence in such young individuals (in her cases ages 12 and 21 years), however, makes one suspect some non-atheromatous process. In describing Russell's studies Hewer wrote that she "does not mention any microscopical abnormality of the cardiac vessels". This is not true. The next major contribution was that of Nadas and his colleagues who first illustrated unequivocal lesions of coronary arteries, which they interpreted cautiously but correctly. ${ }^{6}$ Unfortunately, they did not make it clear that the reported lesions were in the small rather than the large coronary arteries-we have confirmed this finding in the samples that they allowed us to study.

Because of the Nadas report and in view of the 
widely recognised electrocardiographic abnormalities, interpreted by some as representing myocardial ischaemia and even infarction, Boyer et al studied 33 cases of Friedreich's cardiomyopathy for which there was necropsy information on two of the eight that died. ${ }^{7}$ It was clear from their report that they were seeking large coronary lesions of the type often associated with conventional myocardial infarction, and they found coronary atherosclerosis "without lumen occlusion" in one of the two necropsy cases, a 25 year old woman. Based upon clinical evidence, mainly electrocardiograms (there were no coronary arteriograms), they concluded that coronary disease was not the basis of Friedreich's cardiomyopathy. But they did not examine the small coronary arteries in the two necropsy cases, or at least they did not report that they had. Since then the clinical importance of coronary spasm has come to be widely accepted, including its occurrence in Friedreich's cardiomyopathy. ${ }^{29}$ Thus it is possible that some of the "insignificant" lesions in the large coronary arteries may have been erroneously dismissed.

The study reported by Hewer in 1969 is often cited as providing the definitive answer. ${ }^{11}$ From a review of 27 cases he concluded that lesions causing narrowing of the small coronary arteries were indeed present but "that the vast majority of cardiac arteries are not obviously narrowed"; this is a misinterpretation of his own findings to which we will return. He further concluded that the coronary narrowing that he did find was secondary to involution of cardiac muscle, although little evidence is presented to support this interpretation. If he examined any of his sections with the periodic acid-Schiff stain, for example, he does not report it. He does not explain how this unusual and perhaps unique lesion in Friedreich's cardiomyopathy could be secondary to involution of cardiac muscle or how such a process could lead to focal fibromuscular dysplasia or other structural lesions. It is at least as plausible to interpret involution of muscle as being due to coronary disease as the reverse.

But the conduct of the Hewer study itself is more fundamentally flawed. Most subsequent reviewers describing the cardiomyopathy of Friedreich's ataxia refer to his 27 cases, but Hewer was able to examine tissue from only 16 of these cases. Neither the amount or nature of the tissue or its source in the heart is described for these 16 cases and it is uncertain whether he had one slide or more, whether wet tissue was included for special staining, whether the sample came from representative areas of any given cardiac chamber, or how well preserved and suitable for any sort of study some of these archival samples were. He had three (not the often quoted 27) whole hearts to examine, and he made bread loaf slices of them to obtain a large segment of myocardium from which to do his 100 count examinations. Conventional ventricular sections prepared with cuts perpendicular to the epicardium are suitable for examinations of right ventricular or atrial coronary arteries, where most of the small branches run parallel to the epicardium, but not for the left ventricle or interventricular septum where such cuts would lie parallel to the major course of most small coronary arteries. ${ }^{35}$ Though this limited Hewer's sampling opportunity, his subsequent count of 100 consecutively encountered cross sections of arteries does provide the data he sought although it may not be representative of all regions of the heart.

His use of the 100 count approach was then encumbered by the arbitrary criterion that a narrowing was regarded as functionally significant only if $>50 \%$ of the lumen was affected. This makes no allowance for postmortem shrinkage of hearts in which the vessels were not fixed in distension (which would have led to an overestimate of lesions) nor for the possible importance of spasm in diseased arteries with $<50 \%$ narrowing (which would underestimate significance). Most of the coronary branches that regulate flow are of the size being considered (100 to $1000 \mu \mathrm{m}$ in diameter) and observed abnormalities of their walls cannot be dismissed simply because they do not cause $50 \%$ narrowing of the lumen at necropsy.

Small arteries of the heart are not simply passive conduits. To regulate coronary flow they must not only have an adequate lumen but they must also be capable of responding appropriately to neurohormonal signals for dilatation or constriction as metabolic and functional needs require. Structural disease of such vessels may be expected to distort significantly their responses to either neural or hormonal influences, so that they would be unable to dilate or to constrict when they should. Some of the examples we report here (figs 3-6) as well as figs 7 to 9 in Hewer's own study could hardly be expected to behave in a physiological manner, whatever the degree of luminal narrowing may have been.

The most serious error in the Hewer study is his interpretation that $91 \%$ of arteries were normal, or conversely that only $9 \%$ had significantly narrowed lumens. His data do not represent arteries per se, but only cross sections of arteries. What he observed was that $9 \%$ of such cross sections were significantly narrowed, which tells us about only a thin section of a comparatively long artery. In the human left ventricle such arteries are regularly 20 to $30 \mathrm{~mm}$ long, and in the interventricular septum they are often longer. ${ }^{36}$ The sinus node artery is frequently 50 to $100 \mathrm{~mm}$ long in the normal adult human heart. ${ }^{37} \mathrm{~A}$ study with $8 \mu \mathrm{m}$ sections to assess the patency of 
such arteries would require several thousand sections per artery. Even with Hewer's $30 \mu \mathrm{m}$ slices one would need over one thousand sections to assess the patency of such arteries.

An obstructing lesion $(50 \%$ or even $100 \%$ occlusion) need not be very long, but it would eliminate all distal flow in that vessel. If such a lesion were 100 $\mu \mathrm{m}$ long (some of the pronounced narrowings we observed in serial sections were shorter than that), an observer would have only one chance in several hundred sections of a 30 or $40 \mathrm{~mm}$ ( 30000 to $40000 \mu \mathrm{m}$ ) artery of finding such a lesion; Hewer reported that he found lesions in $9 \%$ of the arteries that he examined. This is at least several times more than would be expected by chance alone. We do not dispute the accuracy of his observations which we were able to confirm, but his interpretation is wrong. Hewer in essence made a two dimensional analysis of a three dimensional problem.

In addition to the significant lesions in small coronary arteries we should probably reconsider the findings in the large coronary arteries. Several previous observers have found "atheroma" of major coronary arteries but have regarded them as not clinically significant because the lumen was not obstructed (sic). ${ }^{578}$ We can no longer think of larger coronary arteries as simply passive conduits either. Not only is spasm of the large coronary arteries an accepted clinical entity but it has been found in a patient with Friedreich's cardiomyopathy. ${ }^{29}$ Thus the entire coronary tree must be suspect in the pathogenesis of heart disease in patients with Friedreich's cardiomyopathy.

\section{CARDIONEUROPATHY}

To our knowledge this is the first study of abnormalities of the nerves and ganglia of the heart in Friedreich's ataxia. Extracardiac neural abnormalities (not studied here) may also contribute to disturbances of cardiac rhythm or conduction or repolarisation, as well as contractility and coronary tone. They apparently are responsible for labile hypertension in some patients with Friedreich's ataxia. ${ }^{26}$

One of the most familiar clinical features of Friedreich's cardiomyopathy is widespread inversion of $T$ waves in the electrocardiogram. Possible explanations must include the known focal degeneration and fibrosis in the myocardium, some but certainly not all of which may be related to disease of the small coronary arteries. Another explanation, however, may be neural disease both within and outside the heart. Neural control of the heart profoundly influences almost every component of its function, but it is especially effective upon the processes governing repolarisation and thus the appearance of the
ST segment and $\mathrm{T}$ wave. ${ }^{38}$

Cardioneuropathy may also affect myocardial contractility. The inevitable regional variations in the neural inotropic influence may be the basis of some of the unusual changes in segmental wall motion or other ventricular contractile abnormalities described in Friedreich's cardiomyopathy. ${ }^{13-162027-29}$

\section{CARDIOMYOPATHY}

There is not much dispute about the general nature of the myocardial lesions: hypertrophy of fibres, widely scattered focal degeneration and varying degrees of fibrosis, and the unusual nuclear abnormalities first described by Russell. Hypertrophy of the heart is generalised, as are each of the myocardial lesions. Hypertrophy is sometimes asymmetrical ${ }^{13-15}$; but this is the exception rather than the rule. As discussed previously, ${ }^{8}$ it seems reasonable to attribute some of the focal degeneration and fibrosis in the myocardium to narrowing lesions of the small coronary arteries. The association with focal lesions in the sinus node that we saw in all three of the present cases coupled with focal pronounced narrowing of branches of the sinus node artery is particularly persuasive since all three patients had documented atrial arrhythmias. Neither the pathogenesis nor the functional significance of the bizarre pleomorphic nuclei is apparent.

\section{A MOSAIC CONCEPT OF PATHOGENESIS}

The indisputable lesions of both the small and large coronary arteries, the demonstrable abnormalities of the nerves and ganglia, and the changes seen in the myocytes are three components in a mosaic concept of the pathogenesis of Friedreich's cardiomyopathy (fig 12). All three abnormalities probably have some influence on each other. For example, sufficient narrowing of the arteries-whether by a visualised structural lesion or by intermittent spasm-can cause focal ischaemia not only of myocytes but also of nerves and ganglia. Such focal neural ischaemia may distort responses by the heart to extracardiac neural influence generated either by intermittent

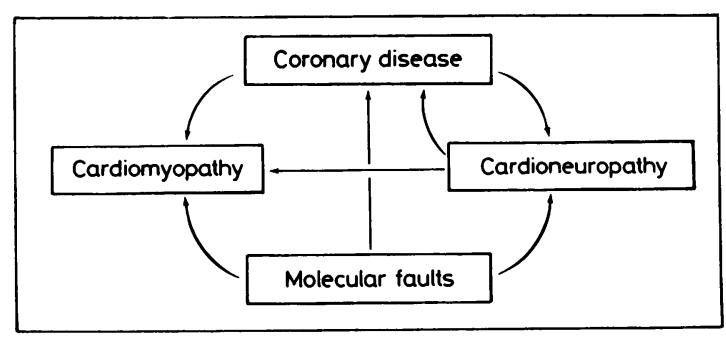

Fig 12 The multifactorial nature of abnormalities in Friedreich's cardiomyopathy. 
reflexes or by more sustained disease of the type intrinsic to Friedreich's ataxia. Neural injury may also be the source of either excitatory ${ }^{39}$ or depressive ${ }^{40}$ cardiogenic reflexes. For example, labile hypertension observed in Friendreich's ataxia ${ }^{27}$ could have its origin within the heart. ${ }^{39}$ From a general assessment of the areas of focal degeneration and fibrosis of myocardium it seems unlikely that these can be entirely attributed to the arterial lesions, but some must be. Further studies in vivo, such as thallium scans and assessment of coronary reserve, are needed to determine how much of the myocardial abnormality is the result of ischaemia, which is largely attributable to the functional behaviour of the small coronary arteries. ${ }^{41-49}$

In one study of 14 patients with Friedreich's cardiomyopathy thallium scans showed abnormal myocardial perfusion in every case. ${ }^{27}$ Although the workers did not interpret their findings as being caused by abnormal coronary flow, claiming that in their own experience the large and small coronary arteries did not show "significant atherosclerotic involvement", their previous morphological study actually demonstrated mild to moderate disease of the small coronary arteries in each of their three cases. ${ }^{12}$ Therriault et al offer no convincing alternative explanation, ${ }^{27}$ but we agree with others ${ }^{42437-49}$ that the most logical cause of functional abnormalities in such thallium scans is abnormal small coronary arteries.

Hypertrophy and myocytopathy of the heart in Friedreich's ataxia can also partially be attributed to a compensatory response to recurring focal ischaemia, but the prevalence of bizarre nuclei within the myocytes suggests some more fundamental fault, perhaps in intracellular metabolism. Whether the cardioneuropathy contributes to myocardial hypertrophy depends upon several factors. If the nerves affected are of sympathetic origin then during any excitatory phase of the disease they may release sufficient local noradrenaline to produce hypertrophy. ${ }^{50} 51$ Later, when the nerves may be destroyed or inactive, the focal loss of a positive inotropic influence may lead to abnormal local stress which could end in compensatory hypertrophy. Overactivity of local nerve endings could even lead to fibromuscular dysplasia of the small or large coronary arteries, as was suggested for the myocardium. If such focal fibromuscular dysplasia has its origin in some non-neural process it may nevertheless make the local area supplied by the affected artery over responsive to either normal or increased neural influence.

All three of these pathogenetic components converge in the cardiac conduction system and particularly the sinus node. There one finds abnormally narrowed arteries, degenerated nerves and ganglia, and pleomorphic nuclei in abundance. Since atrial arrhythmias are common in patients with Friedreich's cardiomyopathy (all three of our cases), this added physiological stress brought about by disease within the conduction system could even be considered a fourth component in the pathogenesis of Friedreich's cardiomyopathy.

Only by concomitantly considering all of the arterial, neural, and myocardial facets of this complex disease can any reasonable synthesis be made of the pathogenesis of Friedreich's cardiomyopathy. In different cases the individual contributions of these components will undoubtedly vary both in size and timing. Ultimately, of course, we must seek further for the various postulated molecular faults which may be responsible, ${ }^{1721-24}$ but it is perplexing how a single or closely related group of such molecular faults could account for such diverse components in this pathogenesis. If there is a common thread, as indeed there may be, it is not yet apparent.

This work was supported in part by the National Heart, Lung and Blood Institute (H-7108 and HL $11,310)$ and by the State of Alabama Program for Research on Sudden Death.

\section{References}

1 Friedreich $\mathrm{N}$. Ueber degenerative atrophie der spinalen hinterstrange. Arch Path Anat Physiol Klin Med 1863;27:1-26.

2 Pentland B, Fox KAA. The heart in Friedreich's ataxia $J$ Neurol Neurosurg Psychiatry 1983;46:1138-42.

3 Child JS, Perloff JK, Bach PM, et al. Cardiac involvement in Friedreich's ataxia: a clinical study of 75 patients. J Am Coll Cardiol 1986;7:1370-8.

4 Laubry C, Heim de Balsac R. A propos des troubles cardiaques de la maladie de Freidreich. Bull Soc Med Hop Paris 1936;52:756-9.

5 Russell DS. Myocarditis in Friedreich's ataxia. J Pathol Bacteriol 1946;58:739-48.

6 Nadas AS, Alimurung MM, Sieracki LA. Cardiac manifestations of Friedreich's ataxia. $N$ Engl J Med 1951;244:239-44.

7 Boyer SH, Chisholm AW, McKusick VA. Cardiac aspects of Friedreich's ataxia. Circulation 1962; 25:493-505.

8 James TN, Fisch C. Observations on the cardiovascular involvement in Friedreich's ataxia. Am Heart $\mathrm{J}$ 1963;66:164-75.

9 Ivemark B, Thoren C. The pathology of the heart in Friedreich's ataxia. Changes in coronary arteries and myocardium. Acta Med Scand 1964;175:227-37.

10 Varnauskas E, Ivemark B, Paulin S, Ryden B. Obscure cardiomyopathies with coronary artery changes. $\mathrm{Am}$ $J$ Cardiol 1967;19:531-41.

11 Hewer RL. The heart in Friedreich's ataxia. Br Heart $J$ 1969;31:5-14. 
12 Lamarche JB, Cote $M$, Lemieux B. The cardiomyopathy of Friedreich's ataxia: morphological observations in 3 cases. Can J Neurol Sci 1980;7:389-96.

13 Gach JV, Andriange M, Franck G. Hypertrophic obstructive cardiomyopathy and Friedreich's ataxia. Am J Cardiol 1971;27:436-41.

14 Boehm TM, Dickerson RB, -Glasser SP. Hypertrophic subaortic stenosis occurring in a patient with Friedreich's ataxia. Am J Med Sci 1971;260:279-84.

15 Ruschhaupt DG, Thilenius OG, Cassels DE. Friedreich's ataxia associated with idiopathic hypertrophic subaortic stenosis. Am Heart $J$ 1972; 84:95-102.

16 Van der Hauwaert LG, Dumoulin M. Hypertrophic cardiomyopathy in Friedreich's ataxia. $\mathrm{Br}$ Heart $\mathrm{J}$ 1976;38:1291-8.

17 Blass JP, Kark RAP, Menon NK. Low activities of the pyruvate and oxoglutarate dehydrogenase complexes in five patients with Friedreich's ataxia. $N$ Engl J Med 1976;295:62-7.

18 O'Brien ET, Dajee H, Ward OC. Friedreich's diseasea family study. Eur J Cardiol 1977;6:15-24.

19 Cote M, Bureau M, Leger C, et al. Evolution of cardiopulmonary involvement in Friedreich's ataxia. Can J Neurol Sci 1979;6:151-7.

20 Berg RA, Kaplan AM, Jarrett PB, Molthan ME. Friedreich's ataxia with acute cardiomyopathy. Am J Dis Child 1980;134:390-3.

21 Barbeau A. Friedreich's ataxia 1980. An overview of the physiopathology. Can J Neurol Sci 1980;7:455-68.

22 Gottdiener JS, Hawley RJ, Maron BJ, Bertorini TF, Engle WK. Characteristics of the cardiac hypertrophy in Friedreich's ataxia. Am Heart $J$ 1982;103:525-31.

23 Stumpf DA. Friedreich's disease: a metabolic cardiomyopathy. Am Heart J 1982;104:887-8.

24 Pasternac A, Wagniart $\mathrm{P}$, Olivenstein $\mathrm{R}$, et al. Increased plasma catecholamines in patients with Friedreich's ataxia. Can J Neurol Sci 1982;9:195-203.

25 Harding AE, Hewer RL. The heart disease of Friedreich's ataxia: a clinical and electrocardiographic study of 115 patients, with an analysis of serial electrocardiographic changes in 30 cases. $Q \mathrm{~J} \mathrm{Med}$ 1983;52:489-502.

26 Margalith D, Dunn HG, Carter JE, Wright JM. Friedreich's ataxia with dysautonomia and labile hypertension. Can J Neurol Sci 1984;11:73-7.

27 Therriault L, Lamoureux G, Cote M, Plourde G, Lemieux B. Cardiomyopathy in Friedreich's ataxia: ventriculography and myocardial imaging with thallium-201. Can J Neurol Sci 1984;11:588-91.

28 Steinsapir K, Lewis W. Dilated cardiomyopathy associated with Friedreich's ataxia. Arch Pathol Lab Med 1985;109:454-6.

29 Sohi GS, Drake C, Flowers NC. Friedreich's ataxia associated with hypertrophic cardiomyopathy and coronary vasospasm. Clin Cardiol 1985;8:537-41.

30 Hawley RJ, Gottdiener JS. Five-year follow-up of Friedreich's ataxia cardiomyopathy. Arch Intern Med 1986;146:483-8.

31 Casazza F, Ferrari F, Finocchiaro G, et al. Echocardiographic evaluation of verapamil in Friedreich's ataxia. Br Heart $J$ 1986;55:400-4.

32 James TN. The sinus node. Am J Cardiol
1977; 40:965-86.

33 James TN. Structure and function of the AV junction. The Mikamo Lecture for 1982. Jpn Circ J 1983;47:1-47.

34 James TN. Primary and secondary cardioneuropathies and their functional significance. $J \mathrm{Am}$ Coll Cardiol 1983;2:983-1002.

35 James TN. Small arteries of the heart. The 36th George E Brown Memorial Lecture. Circulation 1977;56:2-14.

36 James TN, Burch GE. Blood supply of the human interventricular septum. Circulation 1958;17:391-6.

37 James TN. Anatomy of the coronary arteries. Hagerstown, Maryland: Harper and Row, 1961.

38 Abildskov JA. Adrenergic effects on the QT interval of the electrocardiogram. Am Heart $J$ 1976;92:210-6.

39 James TN, Isobe JH, Urthaler F. Analysis of components in a hypertensive cardiogenic chemoreflex. Circulation 1975;52:179-92.

40 Frink RJ, James TH. The intracardiac route of the von Bezold-Jarisch reflex. Am J Physiol 1971;221:1464-9.

41 Strauer BE. Die quantitative bestimmung der koronarreserve zur diagnostik koronarer durchblutungsstorungen. Der Internist 1977;18:579-87.

42 Factor SM, Minase $T$, Cho $S$, Dominitz $R$, Sonnenblick EH. Microvascular spasm in the cardiomyopathic Syrian hamster; a preventable cause of focal myocardial necrosis. Circulation 1982;66: 342-54.

43 Cannon RO III, Watson RM, Rosing DR, Epstein SE. Angina caused by reduced vasodilator reserve of the small coronary arteries. J Am Coll Cardiol 1983;1:1359-73.

44 Opherk D, Mall G, Zebe H, et al. Reduction of coronary reserve: a mechanism for angina pectoris in patients with arterial hypertension and normal coronary arteries. Circulation 1984;69:1-7.

45 Strauer BE. The coronary circulation in hypertensive heart disease. Hypertension 1984;6 (suppl III):74-80.

46 Cannon RO III, Watson RM, Rosing DR, Epstein SE. Efficacy of calcium channel blocker therapy for angina pectoris resulting from small-vessel coronary artery disease and abnormal vasodilator reserve. $A m J$ Cardiol 1985;56:242-6.

47 Kahan A, Nitenberg A, Foult J-M, et al. Decreased coronary reserve in primary scleroderma myocardial disease. Arthritis Rheum 1985;28:637-46.

48 Legrand V, Mancini GBJ, Bates ER, Vogel RA. Evidence of abnormal vasodilator reserve in coronary spasm. Am J Cardiol 1986;57:481-2.

49 Kimball BP, Shurvell BL, Mildenberger RR, Houle S, McLaughlin PR. Abnormal thallium kinetics in postoperative coarctation of the aorta: evidence for diffuse hypertension-induced vascular pathology. J Am Coll Cardiol 1986;7:538-45.

50 Laks MM, Morady F, Swan HJC. Myocardial hypertrophy produced by chronic infusion of subhypertensive doses of norepinephrine in the dog. Chest 1973;64:75-8.

51 Simpson P. Stimulation of hypertrophy of cultured neonatal rat heart cells through an $\alpha_{1}$-adrenergic receptor and induction of beating through an $\alpha_{1}$-and $\beta_{1}$-adrenergic receptor interaction. Circ Res 1985;56:884-94. 